\title{
Musical evolution in the lab exhibits rhythmic universals
}

\author{
Andrea Ravignani ${ }^{1,2 \star}$, Tania Delgado ${ }^{1,3}$ and Simon Kirby ${ }^{1}$
}

\begin{abstract}
Music exhibits some cross-cultural similarities, despite its variety across the world. Evidence from a broad range of human cultures suggests the existence of musical universals', here defined as strong regularities emerging across cultures above chance. In particular, humans demonstrate a general proclivity for rhythm ${ }^{2}$, although little is known about why music is particularly rhythmic and why the same structural regularities are present in rhythms around the world. We empirically investigate the mechanisms underlying musical universals for rhythm, showing how music can evolve culturally from randomness. Human participants were asked to imitate sets of randomly generated drumming sequences and their imitation attempts became the training set for the next participants in independent transmission chains. By perceiving and imitating drumming sequences from each other, participants turned initially random sequences into rhythmically structured patterns. Drumming patterns developed into rhythms that are more structured, easier to learn, distinctive for each experimental cultural tradition and characterized by all six statistical universals found among world music'; the patterns appear to be adapted to human learning, memory and cognition. We conclude that musical rhythm partially arises from the influence of human cognitive and biological biases on the process of cultural evolution ${ }^{3}$.
\end{abstract}

Percussion instruments may have provided the first form of musical expression in human evolution. Great apes, our closest living relatives, show drumming behaviour ${ }^{4}$, which they can learn socially $y^{5}$, producing some human-like rhythmic sequences ${ }^{6}$. Percussive behaviour may therefore have already been present in our ancestors some million years ago, before the split between the human and Pan lineages ${ }^{2}$. Archaeological findings also suggest that the first human musical instrument might have been percussive, as also attested in modern hunter-gatherer societies around the world ${ }^{7}$. This makes rhythm a particularly apt musical dimension for reconstructing crucial steps in the evolution of music.

Six rhythmic features can be considered human universals, showing a greater than chance frequency overall and appearing in all geographic regions of the world. These statistical universals ${ }^{1}$ are:

- A regularly spaced (isochronous) underlying beat, akin to an implicit metronome.

- Hierarchical organization of beats of unequal strength, so that some events in time are marked with respect to others.

- Grouping of beats in two (for example, marches) or three (for example, waltzes).

- A preference for binary (2-beat) groupings.
- Clustering of beat durations around a few values distributed in less than five durational categories.

- The use of durations from different categories to construct riffs, that is, rhythmic motifs or tunes.

Until now, research on musical universals has focused either on individual psychological processes ${ }^{8}$, investigating rhythm perception/production in meticulously controlled environments ${ }^{9,10}$, or on large-scale phenomena, performing cross-cultural analyses of world musical traditions ${ }^{11,12}$. Combining these approaches, we show that basic psychological mechanisms (working memory, perceptual primitives, categorical perception and so on) can lead to large-scale musical universals via cultural transmission. Our experiment aimed to reconstruct, in a laboratory setting (Fig. 1a), how initially unstructured sounds might have been shaped into complex musical systems by early humans perceiving and imitating them ${ }^{7,12,13}$. We tested experimentally controlled human microsocieties and show that cultural transmission accounts for the emergence of both structural regularities and all of the predicted rhythmic universals. Our method builds on previous experimental methodologies, which showed how systematic structure may emerge from weak learning biases ${ }^{14}$. Iterated learning (Fig. 1a) refers to a process by which the individual learns a new behaviour by observing another individual who acquired the behaviour in the same way ${ }^{15}$. This method directly taps into the dynamics of cultural transmission, thereby enabling an empirical approach to human cultural evolution $^{16}$. Iterated learning of artificial sounds ${ }^{17}$, visual representations ${ }^{18}$ and language-like systems $\mathrm{s}^{15,19} \mathrm{can}$ lead to a large range of outcomes. However, two characteristics seem to emerge in most experiments: random patterns evolve into sequences that exhibit increasing learnability and structure over generations of learners ${ }^{19,20}$.

In a similar way to vertical transmission shaping the complexity and variety of musical cultures ${ }^{3,12}$, in our experiment, each participant heard and had to imitate drumming patterns received from a previous participant, who themself had copied them from someone else and thereby potentially introduced errors. In measuring the changes that occurred to the drum patterns, we observed how cognitive biases for rhythm are magnified and mirrored in musical structure and how initially independently reproduced sequences come to pattern together as part of an overall rhythmical system ${ }^{21}$. As predicted, after several experimental generations, initially random sequences transformed into increasingly structured and learnable music-like patterns. In addition, these patterns showed convergence towards all of the six rhythmic universals found in human musical cultures ${ }^{1}$.

First, the sequences acquire systematic structure. Systematicity is a measure of mutual predictability among the elements of a system;

${ }^{1}$ Centre for Language Evolution, School of Philosophy, Psychology and Language Sciences, University of Edinburgh, Edinburgh EH8 9AD, UK.

${ }^{2}$ Artificial Intelligence Lab, Vrije Universiteit Brussel, Brussels 1050, Belgium. ${ }^{3}$ Department of Cognitive Science, University of California, San Diego, 9500

Gilman Drive, La Jolla, California 92093-0515, USA. *e-mail: andrea.ravignani@gmail.com 


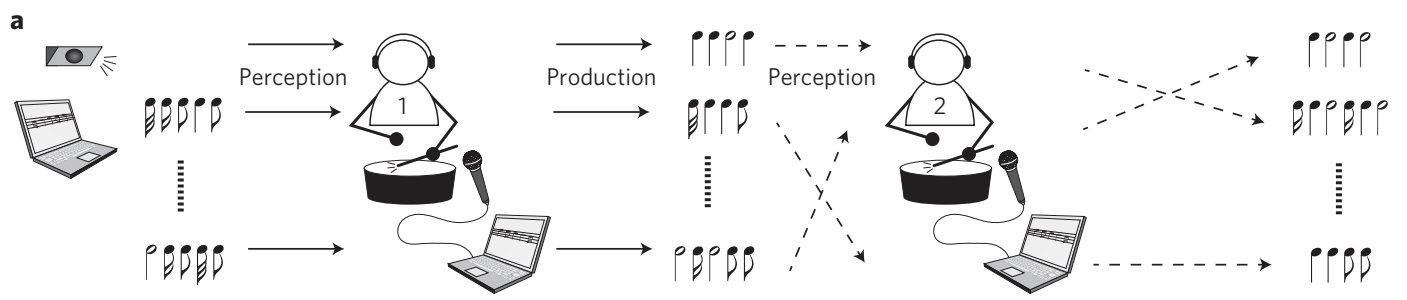

b
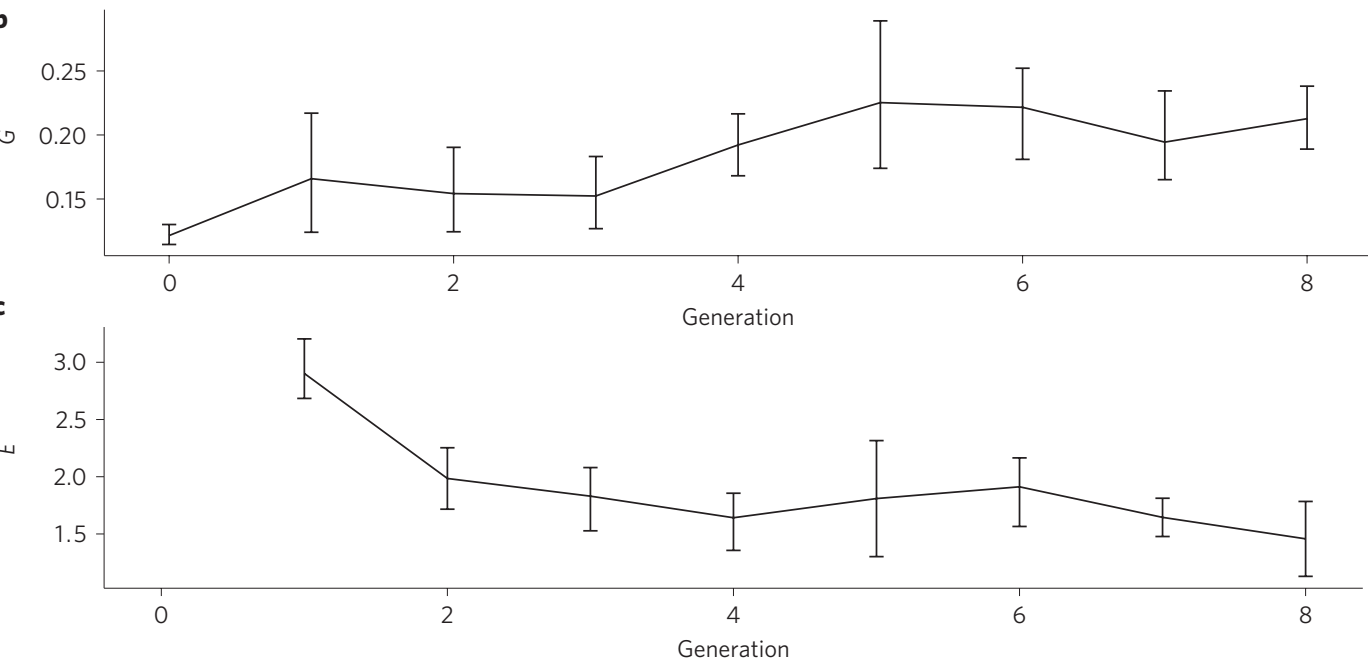

Figure 1 | Cultural transmission over generations by iterated learning. a, The first two transmission steps in a chain of drummers. We generated sequences of drumming patterns with random velocity (hit strength) and time between hits. These random sequences (random generation 0 , leftmost note sequences) sounded completely arrhythmic and mimicked incidental occurrences of sound sequences, either naturally produced or humangenerated, that an early music-less hominid might have attended to. We then presented 32 of these random sequences to an experimental participant (generation 1), who was asked to faithfully copy the rhythm on a drum set immediately after each of the 32 presentations. The sequences thereby produced, with all their copying errors, formed the set of drum patterns presented to the next participant in random order (generation 2 ). This process was repeated until the chain reached 8 participants. To control for the effects of the initial random patterns or particular participants in a chain, the experiment was repeated in six independent chains (not shown), totalling 48 participants. b, The increase in structure/systematicity measure $G$, corresponding to a modified measure of entropy. c, The decrease in imitation errors $E$, equivalent to an increase in learnability/imitation fidelity. Error bars represent bootstrapped $95 \%$ confidence intervals across chains.

it quantifies how much structural information about a whole system is provided by each constituent element. In musical harmony, for instance, rock and roll is very systematic, because knowing a musical excerpt provides a better than chance guess of the chord progressions of a broad range of songs, while dodecaphonic music is less systematic. We found an increase in structural similarities and combinatorial structure over generations (Page's trend test; $L=1558.0$, $m=6, n=9, P<0.001$; Fig. 1b).

Second, sequences become easier to learn. A system or structure is highly learnable if it can be rapidly acquired with low error by an organism. Reproduction errors (time distance between participants' outputs) decrease over generations (Page's trend test; $L=833$, $m=6, n=8, P<0.0001$; Fig. 1c). Learners in later generations found the rhythms easier to imitate accurately, indicating that patterns increasingly fit participants' cognitive biases.

Third, timing patterns converge to durational categories. The frequency distributions of inter-onset intervals (IOIs; the time between consecutive drum hits) of all chains showed a similar pattern across experimental generations: initial uniform distributions (the random patterns presented to the first generation) converged on chain-specific clusters of IOIs by the final generation (Fig. 2). A $K$-means cluster algorithm showed that rhythmic patterns converged to three durational categories (Supplementary Table 1), matching the statistical universal across world musical cultures, which predicts less than five categories ${ }^{1,11}$. The range of durations produced by our participants was consistent with musical rhythms, as used in rhythm experiments. The first cluster in all chains had a median of $203 \mathrm{~ms}$ (Supplementary Table 1), close to $200 \mathrm{~ms}$, a recurrent durational value in musical rhythm and metre ${ }^{22}$. Moreover, the resulting centroids of the clusters were related by numbers close to integer ratios (Supplementary Table 1).

Fourth, systematicity and learnability increase, translating to the emergence of repeating structures (phase-space plots in Fig. 3a). Specifically: (i) rhythmic patterns acquired motivic structure (another musical universal ${ }^{1}$ ) whereby rhythmic 'riffs' emerged, corresponding to polygons in phase-space coordinates where the number of vertices equals the length of the repeating riff within a pattern ${ }^{23}$; (ii) riffs were used multiple times by each participant across separate drum patterns, shown by similar polygons overlapping in one phase-space plot; (iii) motivic patterns evolved gradually as they were passed from earlier to later generations (Fig. 3a, similar polygons in different plots of one chain); and (iv) riffs partly differed between chains (different polygons in different chains).

Fifth, sequences become more metronomic (isochronous), hierarchically structured (metrical) and composed through durations that are related by small-integer ratios. Isochrony and metre in perceived music are usually probed by asking participants to tap along, testing whether their taps occur at simple multiples or at divisors of the occurring musical intervals. As our task involved musical production, we reversed the above logic. Participants creating a metrical grid with binary and ternary subdivisions and an underlying regular beat ${ }^{24}$ would produce: (i) adjacent IOIs related by small integer ratios; (ii) IOIs with many values close to 1:1 (equal-length IOIs); (iii) IOIs with ratios of two and three 


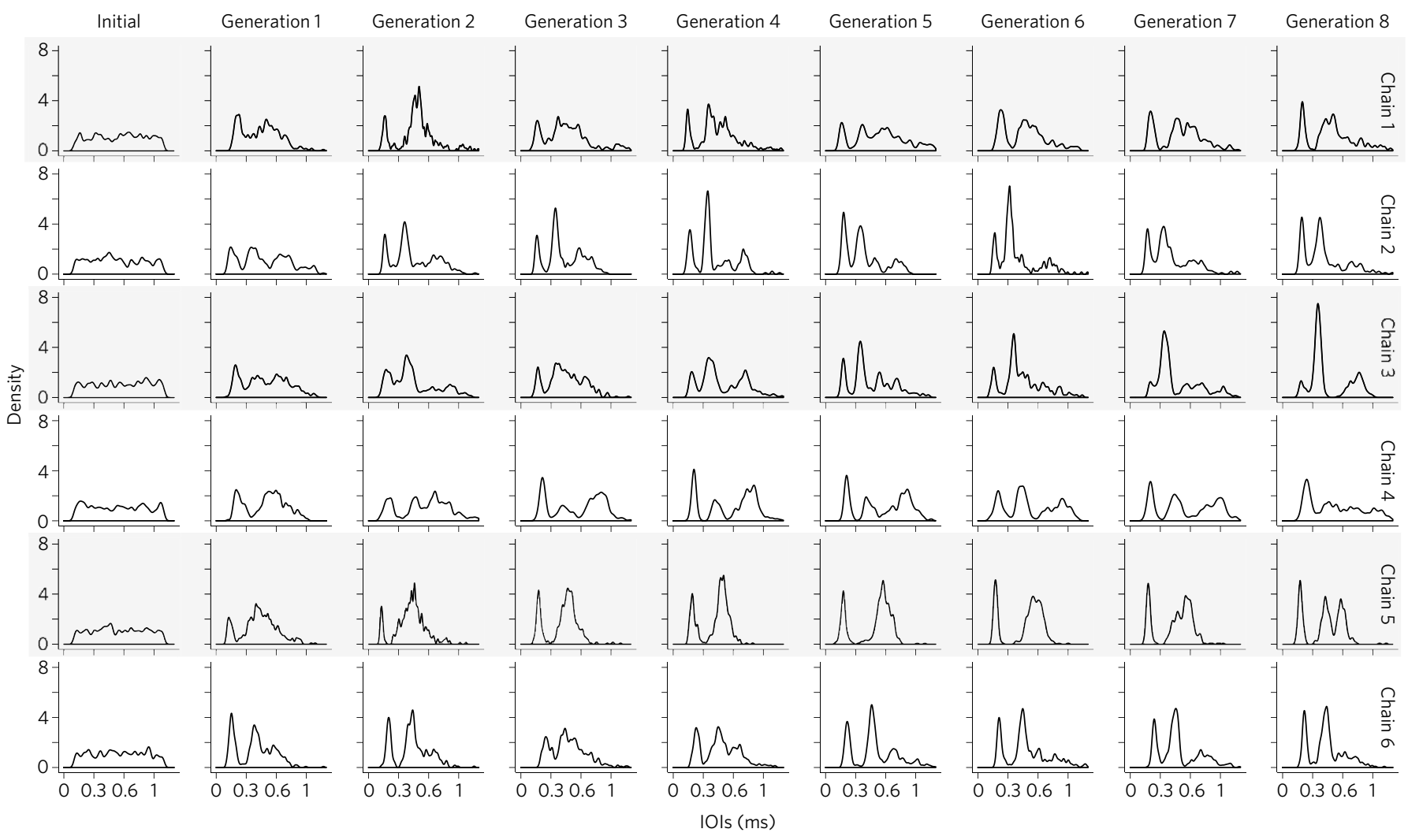

Figure $\mathbf{2}$ | Frequency distributions of IOls in drumming sequences for each chain (rows) and generation (columns). IOls were pooled across all 32 patterns and plotted using kernel density estimations (KDEs; density expressed in arbitrary units). Over the course of generations, there is a gradual development of interval durations, which become more categorical (corresponding to more peaked distributions) towards the last generation (rightmost column). From left to right, the figure shows how each chain slowly converges towards a different distribution of IOls from the other chains upon the final generation. Centroids of each of the distributions of the last generations can be found in the Supplementary Information. Extreme data points, corresponding to values $>1.1 \mathrm{~s}$, and representing $<5 \%$ of all data, are not shown.

(showing binary and ternary subdivisions) ${ }^{24}$; and (iv) the strongest beats at IOIs twice or three times their multiples of each other, suggesting musical metre. We found that the distributions of ratios in the last generation (Fig. 4a,b) significantly differed from a simulated uniform ratio distribution, predicted under null hypothesis of no pairwise structure between IOIs (two-sample KolmogorovSmirnov test, all $D>0.08, P<0.01$; see Supplementary Information). This holds for both the distributions of adjacent IOIs and of IOIs between high-intensity hits, suggesting the existence of structural relationships between IOIs. We then tested whether peaks in the ratio distributions (Fig. 4a,b) corresponded to specific constant relationships between IOIs (see Methods). One of the highest peaks in Fig. $4 \mathrm{a}$ occurs at 1.015, and the median of the distribution is at 0.968 . Both values are close to $1: 1$, providing some evidence for isochrony, another universal. We then tested whether the highest peaks in Fig. 4a,b coincided beyond chance with those expected theoretically in actual music. For adjacent ratios, we found four peaks, at 1:2, 1:4, 3:2 and 3:4. The match between ratios expected in music and experimental ratios is not attributable to chance. (The corresponding Jaccard index, which measures overlap ${ }^{25}$, is $J=0.222$; a randomization test returned an average $J$ value of 0.064 , with a pseudo $P$ value $\left(P^{\prime}\right)$ of 0.029 ; see Supplementary Information.) A similar analysis on the distribution of the ratios of IOIs between strong beats (median $=0.947$ ) found support for the hypothesis that metre is exclusively binary $\left(J=0.028, P^{\prime}=0.045\right)$, with strong and weak alternating beats, but not exclusively ternary $(J=.028$, $\left.P^{\prime}=1.0\right)$. Strong beats occurred above chance in intervals that were half or double each other in length (that is, related by $1: 2$ and 2:1 ratios). Notes of ternary length existed, but did not always coincide with the metrical grid (for example, a binary metre with many notes of length $1 / 4$ and 3/4). This suggests the presence of: (i) an underlying regular beat; which is (ii) composed of alternating weak-strong beats; and (iii) used as a reference duration to generate the duration of other notes (by multiplying and dividing it by two or three), providing evidence for the remaining universals.

Sixth, chains evolve independently. We calculated the KolmogorovSmirnov $D$ statistic for each generation and pairs of participants using their distribution of IOIs to quantify the degree of cultural divergence. Chains significantly diverged over generations towards separate lineages, with different timing structures $(L=1586.0, m=6, n=9$, $P<0.001$; Fig. 3b). Moreover, all IOI distributions of the final generations were significantly different between chain pairs (all $D<0.3$, all $P<0.01$; Supplementary Table 2). Hence, the drum patterns within the same lineage participated in a system of rhythmic patterns, sharing similar characteristics or motifs. As in actual music ${ }^{12}$, chains gained more structure over generations, although each transmission chain developed its own set of structural features.

It has been debated whether some human biological traits evolved under selective pressures to specifically hear and perform music $c^{2,7,26-28}$. Our data support an alternative hypothesis-that musical structure appears to evolve out of, and is shaped by, more general constraints on learning and memory. In this experiment, rhythmic features evolved cumulatively and gradually from randomness. We obtained divergent musical cultures, where each 'musical culture' (corresponding to an experimental chain) constituted a system by itself. The transmission process we recreated in the lab led to the appearance of design; the patterns evolved in such a way that they appeared to be well adapted to the challenge of being 
a
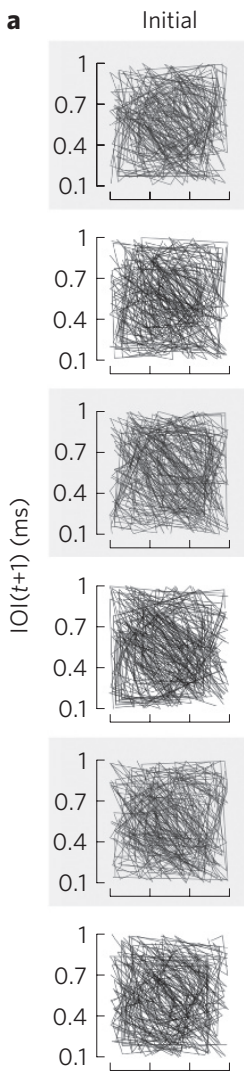

0.10 .40 .71
Generation 1
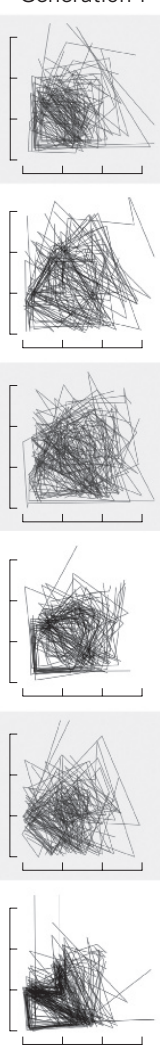

$0.10 .40 .7 \quad 1$
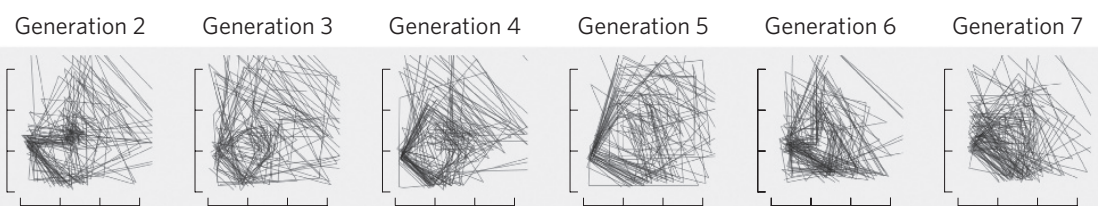

Generation 8
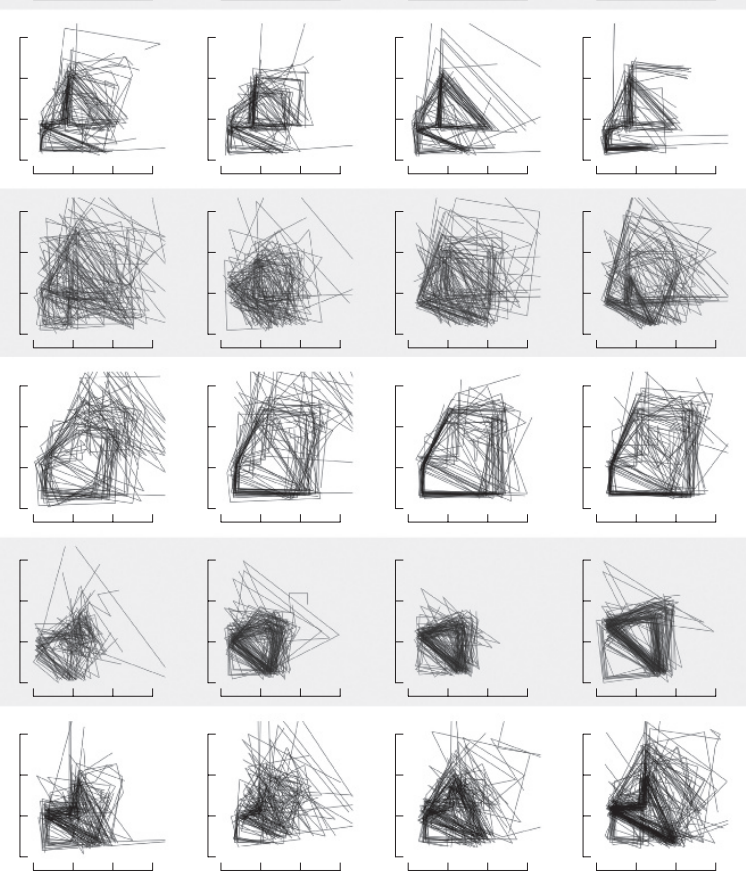

$0.10 .40 .7 \quad 1$
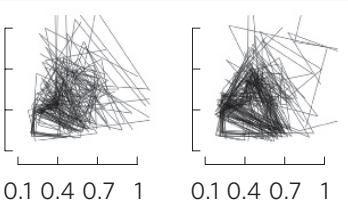

$\mathrm{IOI}(t)(\mathrm{ms})$

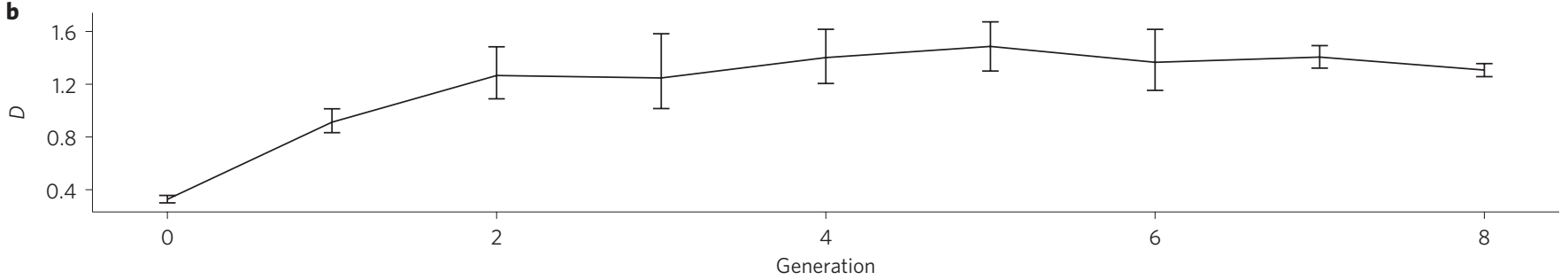

Figure 3 | Emergence of rhythmic riffs and cultural specificity. a, Phase-space diagrams of all chains (top to bottom) and generations (left to right). Each phase-space diagram depicts one participant's output (all 32 patterns). In a phase-space diagram, the axes denote the state of a variable at consecutive points in time, $t$ and $t+1$. The duration of each note ( $x$-axis) is plotted against the duration of the next note ( $y$-axis) as a dot and consecutive dots are joined by a line. This is repeated for all 32 patterns. The phase-space plots here show the evolution of patterns of length $\geq 3$, with increasing regularities over generations ${ }^{23}$. Closed polygons represent repeating drumming patterns. For instance, chain 5 shows a clear emergence (already by the third generation) of a repeating pattern of length 3 , illustrated by a triangle. Chain 3 converges instead towards drumming patterns containing a combination of two similar ternary patterns, inferred by the two non-overlapping triangles. Chain 2 converges towards patterns including a non-repeating sequence of length 5, deduced by the 5-edged segmented line. Also, notice how the vertices of the polygons map to the centroids found with the $K$-means clustering algorithm (Supplementary Information); for example, the centroids of chain 5 are at 177,436 and 665 ms, while those of chain 3 are at 202 , 355 and $764 \mathrm{~ms}$. These approximate values can be found when examining and comparing last generations' phase-space plots of chains 3 and 5 . $\mathbf{b}$, The rise of divergence measure $D$ across chains. Over generations, variability between chains increases. This, together with the increase in $G$, suggests that a distinct yet systematic musical culture emerges in each chain. Error bars represent bootstrapped $95 \%$ confidence intervals across chains.

learnable. Generation after generation, learners introduced errors in their efforts to replicate the sequences they heard. The process eventually resulted in the emergence of rhythmic patterns that were easier to reproduce. Systematic similarities between patterns emerged within a chain; patterns that no longer act independently may facilitate learning over generations, as it is easier to remember a small number of motifs rather than thirty-two totally independent patterns. The participants chosen were non-musicians, so no existing skills in music performance could account for the quick generation of the musical patterns we observed. They were instructed to recreate each sequence as closely as possible and not to innovate or treat the sequences as being related. Crucially, as in human music, our laboratory experiment led to the emergence of commonalities, but also diversity. This experiment provides evidence for the universality of musical features emerging through cultural transmission ${ }^{1,3}$.

Similarly to previous results on the evolution of linguistic structure ${ }^{15,17,19}$, we hypothesize that a few perceptual, learning and production biases may be responsible for the regularities that evolved in our drumming patterns. The formation of durational categories and small-integer ratios between intervals might be partially amenable to the categorical perception of rhythmic sequences. In fact, small 

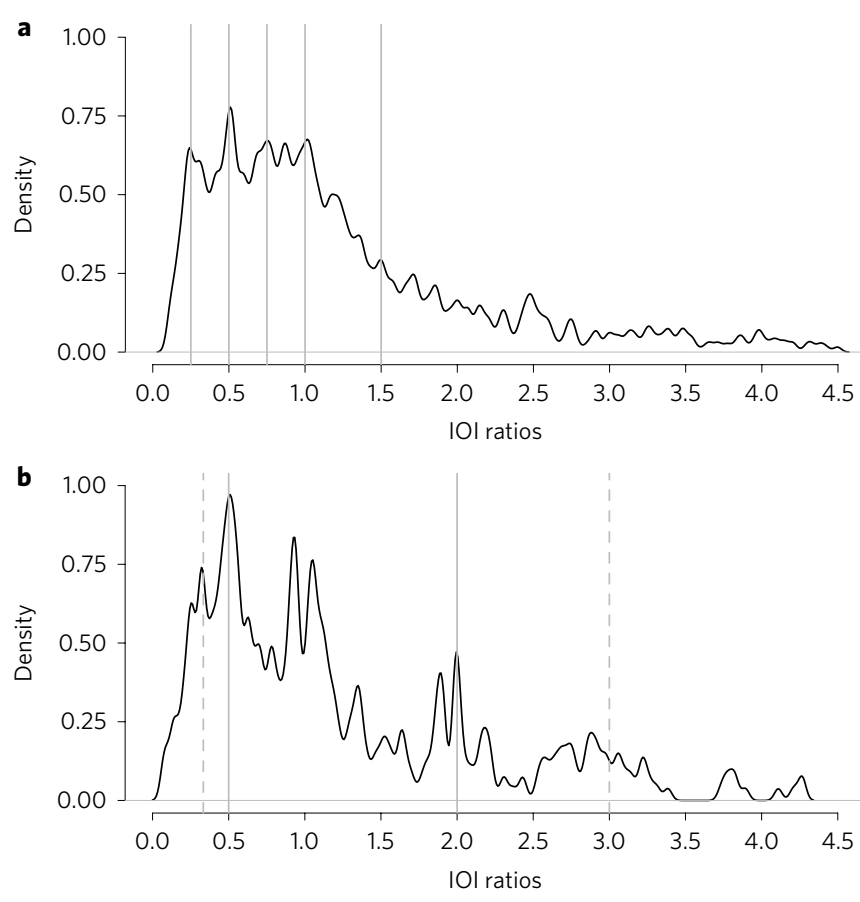

Figure 4 | Statistical universals in durational patterns. a, Frequency distributions of the ratios between adjacent IOIs, pooled across all 32 patterns produced by the six participants in the last generation, calculated using KDEs (density normalized to 1). For each pattern, we calculated the ratios between all adjacent IOIs in that pattern $\left(\mathrm{INI}_{1} / \mathrm{INI}_{2}, \mathrm{INI}_{2} / \mathrm{INI}_{3}, \ldots\right)$. The distribution shows peaks (local maxima) centred at 1:2, 1:1, 1:3, 2:3 and 5:2 (solid lines). b, Frequency distribution of ratios of durations between the $50 \%$ strongest beats (all drum hits above the median hit strength) within a pattern, pooled across all last-generation participants. The solid lines represent the 1:2 and 2:1 (binary) ratios, while the dashed lines represent the 1:3 and 3:1 (ternary) ratios. While several hypothesized ratios emerged as peaks in the distribution (for example, 1:2 and 2:1), there are also peaks that do not map to precise integer ratios, which are attributable to a number of potential factors (cultural, experimental and so on). Extreme data points that correspond to values $>4.5$ and represent $<5 \%$ of all data, are not shown.

ratios function as attractors when musicians are asked to categorize notes of varying durations that are not related by integer ratios ${ }^{29}$. The proximity, although not equality, to integer ratios dovetails with previous findings in music psychology $\mathrm{y}^{30}$. The emergence of a few durational categories and motifs may instead be a by-product of the human tendency to compress sensory stimuli, possibly dictated by working memory constraints and a limited capacity for processing information ${ }^{20}$. Conversely, motor biases seem to only moderately influence the structures obtained; humans have a preferred tapping rate ref $^{22} 600 \mathrm{~ms}$ on average, which was rarely found in our IOI distributions and clusters (Supplementary Table 1 and Fig. 2). However, our experiment cannot disentangle which of the human biases generating musical features are basic and which are acquired, and at least two alternative hypotheses can account for our results. In other words, the fact that our participants had already been exposed to a musical culture may have shaped the results. However, two points counteract this interpretation. First, we saw clear divergence between chains, suggesting that there was no single culturally acquired attractor that was driving the evolution of the systems. Second, there were striking parallels in the evolution of systematic structure between this experiment and another sequence learning experiment in the non-musical domain ${ }^{21}$. Ultimately, cross-cultural replications of this experiment are needed to accurately gauge the influence of acquired biases in this task.

Music, language and dance all involve copying to some extent, although imitation/copying is only one of many factors in their evolution $^{3,18,27,28}$. Although the motivations to copy probably differ, the outcomes seem to be similar. We believe the assumption that early humans might have had a motivation to copy music-like sequences is quite realistic. Several hypotheses on the origins of the biological capacity for musical rhythm involve some motivation to copy or imitate. These hypotheses often suggest imitation, learning or synchronization of audio-motor behaviours as necessary steps to achieve interindividual coordination, group cohesiveness, mating success or territorial defence, providing in turn evolutionary pressures on the development of rhythmic abilities in modern humans ${ }^{26}$.

Human music is inherently structured, showing a few structural similarities across musical cultures and traditions. Why do these similarities arise? How do different musical traditions end up with similar features? We have addressed these questions empirically: in the laboratory, we set the conditions for random percussion patterns to be transmitted, similarly to real musical traditions. As a result, we were able to witness the evolution of musical rhythmic structure in real time, as it responded to human constraints and converged towards all six statistical universals found among world rhythms. Musical rhythmic universals arise because human behaviour and cognition slightly transform what is copied ${ }^{13,15,18,19}$. These transformations, amplified by the process of cultural transmission, lead to diverse musical traditions that contain nonetheless a few universals: traces of the biology of the organisms who created them.

\section{Methods}

Participants. Forty-eight participants (mean age 23 years 4 months; females $=37$ ) were recruited from the University of Edinburgh's graduate employment service "to participate in a 30 -minute drumming experiment". Each received $£ 5$ for participating. Musicians (those that had formal musical training or regularly practiced a musical instrument) were excluded from participation. The sample size was established a priori on the basis of a meta-analysis of previous iterated learning experiments ${ }^{14,17,31-36}$.

This experiment was modelled on a simple transmission chain paradigm, in which learners received training inputs from the outputs of the previous learners ${ }^{16}$. Participants were randomly assigned to six different lineages (transmission chains: 1-6), each containing eight 'generations' of learners (1-8). The first generation of participants in all chains heard different randomly generated patterns as training input (first column in Fig. 2 and Fig. 3a).

Stimuli. Participants in each generation were presented with 32 drum patterns. These patterns were either random drum sequences (generation 1 ) or sequences produced by a previous participant (generations $2-8$ ). The 32 initial and independent drum patterns were each composed of 12 MIDI (Musical Instrument Digital Interface) snare drum hits (Supplementary Information). Each chain had its own unique set of 32 initial random patterns. Each snare drum hit in the initial sequences had a random velocity (force and speed used to play an instrument) and the IOIs (duration between the start of one note and the start of the next note) were random. An additional cymbal sound was always presented $1.5 \mathrm{~s}$ after the last snare hit and signalled the end of a sequence. The cymbal timing was neither counted as part of the pattern nor included in the analyses. Participants heard and reproduced two blocks of the same 32 drum patterns, with the order of drum patterns within each block randomized. The first block of patterns was intended for the participant to practice drumming and copying. Patterns reproduced in the second block, recorded on a laptop, were used as the training stimuli for the next learner in the chain.

Procedure. Participants were given headphones, a single drumstick and an Alesis SamplePad, which was connected to a Macbook Pro laptop via a Duo-Capture EX USB-MIDI interface. The Python code that recorded the drumming patterns rounded temporal information to the nearest millisecond (although the theoretical maximum resolution of MIDI is slightly better than $1 \mathrm{~ms}$ ). The interface had four independent drum pads; three produced the snare drum sound and one produced the cymbal sound, which participants struck to conclude a pattern. Participants were instructed to reproduce each pattern immediately after hearing it to the best of their ability. Each sequence was recorded and given to the next participant in the chain. Participants were unaware that they would be listening to stimuli produced by a previous learner. After the behavioural task, participants completed a questionnaire (Supplementary Information). 
Analyses. The output patterns were analysed to determine if the the initially independent sequences evolved, becoming easier to learn over generations and forming rhythmic-like systems with structural regularities. Data analysis was performed in R, Stata 11.0 and using custom written Python scripts. All analyses were performed on the IOI between contiguous drum hits within a pattern. Experiments in human perception of musical rhythms have shown that the IOI is usually more important than the length of the notes themselves ${ }^{37}$. Several quantitative measures were adapted to assess the learnability and structure of the patterns ${ }^{17,20,38-46}$.

Beat and metre. Ratios were taken to normalize with respect to tempo and to compare structures (rather than absolute durations) across patterns. For each ratio distribution, we found the location of the maxima by taking the second derivative of the kernel density estimation (KDE) function. We then tested whether these fixed IOI relationships (the peaks in Fig. 2) coincided beyond chance with those expected theoretically. The most parsimonious way of generating a musical duration from another is to multiply or divide it by two, three or four. Hence we predicted that we would find, with high frequency, ratios of 1:1 (equal duration IOIs), $1: 2,1: 3,1: 4,2: 3,3: 4$, and their reciprocals, giving a total of 11 expected theoretical ratios. As the predicted ratios spanned 11 possible values, we extracted the 11 most frequent ratios from our empirical distributions. We then matched the expected with the empirical ratios (with a 0.01 tolerance on ratio differences) and quantified the match using the Jaccard index ${ }^{25}$. Given two sets, the Jaccard index is calculated as the ratio between their union and their intersection, that is, the number of elements in common divided by the number of overall elements. Finally, we performed a Monte Carlo simulation with 1 million iterations to test whether the matching of the predicted and found peaks was attributable to chance. This provided a $P^{\prime}$ value, calculated as the proportion of randomizations with an average Jaccard index greater than or equal to the empirical Jaccard index; that is, the relative number of cases for which a list of 11 random ratios has equal number or more matches with predicted ratios than the 11 empirical ratios.

Structure and systematicity. Unlike previous cultural transmission research, the transmitted behaviour in this experiment was continuous (that is, time intervals) rather than discrete. We discretized the intervals into three categories using a $K$-means clustering algorithm (Supplementary Table 1 ). We mapped each duration to the tercile it belonged to (for example, three durations such as $(0.1,0.8,0.4 \mathrm{~ms}$ ) would map to (short, long, medium)). The number of categories in the $K$-means algorithm was established using the Elbow method ${ }^{47}$, with three categories emerging as the most parsimonious clustering for each chain (Supplementary Information). We then calculated a grammatical structure index ( $G$, a modified measure for entropy that is comparable with previous studies ${ }^{41}$ ) for each participant.

Learnabilty. The decrease in imitation errors $(E)$ is equivalent to an increase in learnability/imitation fidelity. We calculated this as the (edit) time distance between two drum patterns; that is, the total cost of the minimal cost set of substitutions, insertions or deletions among IOIs necessary to transform the pattern of durations a participant has heard into the pattern they have reproduced, where the edit costs are taken to be the absolute difference in time between durations ${ }^{43}$. The time distance between identical patterns equals zero. Notice that, unlike other metrics in musicology that assume beat induction or metrical hierarchies ${ }^{48,49}$, this edit distance minimizes assumptions about metrical, top-down processing.

Code availability. Data analysis was performed in R, Stata 11.0 and using custom written Python scripts. All scripts are available from the corresponding author

Data availability. The data that support the findings of this study are available for download as supplementary material.

Received 5 June 2016; accepted 12 October 2016; published 19 December 2016

\section{References}

1. Savage, P. E., Brown, S., Sakai, E. \& Currie, T. E. Statistical universals reveal the structures and functions of human music. Proc. Natl Acad. Sci. USA 112, 8987-8992 (2015)

2. Fitch, W. T. in Language and Music as Cognitive Systems (eds Rebuschat, P. et al.) ch. 11, 73-95 (Oxford Univ. Press, 2009).

3. Trehub, S. E. Cross-cultural convergence of musical features. Proc. Natl Acad. Sci. USA 112, 8809-8810 (2015).

4. Ravignani, A. et al. Primate drum kit: a system for studying acoustic pattern production by non-human primates using acceleration and strain sensors. Sensors 13, 9790-9820 (2013).

5. Fuhrmann, D., Ravignani, A., Marshall-Pescini, S. \& Whiten, A. Synchrony and motor mimicking in chimpanzee observational learning. Sci. Rep. 4, 5283 (2014).
6. Dufour, V., Poulin, N., Curé, C. \& Sterck, E. H. Chimpanzee drumming: a spontaneous performance with characteristics of human musical drumming. Sci. Rep. 5, 11320 (2015).

7. Morley, I. The Evolutionary Origins and Archaeology of Music $\mathrm{PhD}$ thesis, Cambridge Univ. (2003).

8. Fritz, T. et al. Universal recognition of three basic emotions in music. Curr. Biol. 19, 573-576 (2009).

9. Repp, B. H., Iversen, J. R. \& Patel, A. D. Tracking an imposed beat within a metrical grid. Music Percept. 26, 1-18 (2008).

10. Winkler, I., Háden, G. P., Ladinig, O., Sziller, I. \& Honing, H. Newborn infants detect the beat in music. Proc. Natl Acad. Sci. USA 106, 2468-2471 (2009).

11. Brown, S. \& Jordania, J. Universals in the world's musics. Psychol. Music 41, 229-248 (2013).

12. Le Bomin, S., Lecointre, G. \& Heyer, E. The evolution of musical diversity: the key role of vertical transmission. PloS ONE 11, e0151570 (2016).

13. Morgan, T. et al. Experimental evidence for the co-evolution of hominin tool-making teaching and language. Nat. Commun. 6, 6029 (2015).

14. Kirby, S., Dowman, M. \& Griffiths, T. L. Innateness and culture in the evolution of language. Proc. Natl Acad. Sci. USA 104, 5241-5245 (2007).

15. Kirby, S. \& Hurford, J. R. in Simulating the Evolution of Language (eds Cangelosi, A. \& Parisi, D.) 121-147 (Springer, 2002).

16. Mesoudi, A. \& Whiten, A. The multiple roles of cultural transmission experiments in understanding human cultural evolution. Phil. Trans. R. Soc. B 363, 3489-3501 (2008).

17. Verhoef, T., Kirby, S. \& de Boer, B. Emergence of combinatorial structure and economy through iterated learning with continuous acoustic signals. J. Phonetics 43, 57-68 (2014).

18. Tamariz, M. \& Kirby, S. Culture: copying, compression, and conventionality. Cogn. Sci. 39, 171-183 (2015).

19. Kirby, S., Cornish, H. \& Smith, K. Cumulative cultural evolution in the laboratory: an experimental approach to the origins of structure in human language. Proc. Natl Acad. Sci. USA 105, 10681-10686 (2008).

20. Kirby, S., Tamariz, M., Cornish, H. \& Smith, K. Compression and communication in the cultural evolution of linguistic structure. Cognition 141, 87-102 (2015)

21. Cornish, H., Smith, K. \& Kirby, S. Systems from sequences: an iterated learning account of the emergence of systematic structure in a non-linguistic task. In Proc. 35th Annual Conf. Cognitive Science Soc. (eds Knauff, M. et al.) 340-345 (Cognitive Science Society, 2013).

22. London, J. Hearing in Time: Psychological Aspects of Musical Meter (Oxford Univ. Press, 2012).

23. Ravignani, A. Visualizing and interpreting rhythmic patterns using phase space plots. Music Percept. (in the press).

24. Celma-Miralles, A., de Menezes, R. F. \& Toro, J. M. Look at the beat, feel the meter: top-down effects of meter induction on auditory and visual modalities. Front. Hum. Neurosci. 10, 108 (2016).

25. Jaccard, P. The distribution of the flora in the alpine zone. New Phytol. 11, 37-50 (1912)

26. Iversen, J. R. in The Cambridge Companion to Percussion (ed. Hartenberger, R.) Ch. 21, 267-280 (Cambridge Univ. Press, 2016).

27. Laland, K., Wilkins, C. \& Clayton, N. The evolution of dance. Curr. Biol. 26, R5-R9 (2016).

28. Ravignani, A. \& Cook, P. The evolutionary biology of dance without frills. Curr. Biol. 26, R878-R879 (2016).

29. Desain, P. \& Honing, H. The formation of rhythmic categories and metric priming. Perception 32, 341-365 (2002).

30. Repp, B. H., London, J. \& Keller, P. E. Systematic distortions in musicians: reproduction of cyclic three-interval rhythms. Music Percept. 30, 291-305 (2013).

31. Smith, K. \& Wonnacott, E. Eliminating unpredictable variation through iterated learning. Cognition 116, 444-449 (2010).

32. Kalish, M. L., Griffiths, T. L. \& Lewandowsky, S. Iterated learning: intergenerational knowledge transmission reveals inductive biases. Psychon. Bull. Rev. 14, 288-294 (2007).

33. Verhoef, T. The origins of duality of patterning in artificial whistled languages. Lang. Cogn. 4, 357-380 (2012).

34. Garrod, S., Fay, N., Rogers, S., Walker, B. \& Swoboda, N. Can iterated learning explain the emergence of graphical symbols? Interact. Stud. 11, 33-50 (2010)

35. Kempe, V., Gauvrit, N. \& Forsyth, D. Structure emerges faster during cultural transmission in children than in adults. Cognition 136, 247-254 (2015).

36. Silvey, C., Kirby, S. \& Smith, K. Word meanings evolve to selectively preserve distinctions on salient dimensions. Cogn. Sci. 39, 212-226 (2015).

37. Parncutt, R. A perceptual model of pulse salience and metrical accent in musical rhythms. Music Percept. 11, 409-464 (1994).

38. Boon, J. P. \& Decroly, O. Dynamical systems theory for music dynamics. Chaos 5, 501-508 (1995). 
39. Caldwell, C. A. \& Millen, A. E. Studying cumulative cultural evolution in the laboratory. Phil. Trans. R. Soc. B 363, 3529-3539 (2008).

40. Clayton, M. R. Observing entrainment in music performance: video-based observational analysis of Indian musicians' tanpura playing and beat marking. Music. Sci. 11, 27-59 (2007).

41. Jamieson, R. K. \& Mewhort, D. Applying an exemplar model to the artificial-grammar task: inferring grammaticality from similarity. Q. J. Exp. Psychol. 62, 550-575 (2009).

42. Kirby, S., Griffiths, T. \& Smith, K. Iterated learning and the evolution of language. Curr. Opin. Neurobiol. 28, 108-114 (2014).

43. Levenshtein, V. I. Binary codes capable of correcting deletions, insertions, and reversals. Sov. Physi. Doklady 10, 707-710 (1966).

44. Matthews, C., Roberts, G. \& Caldwell, C. A. Opportunity to assimilate and pressure to discriminate can generate cultural divergence in the laboratory. Evol. Hum. Behav. 33, 759-770 (2012).

45. Ravignani, A. \& Sonnweber, R. Measuring teaching through hormones and time series analysis: towards a comparative framework. Behav. Brain Sci. 38, 40-41 (2015).

46. Jadoul, Y., Ravignani, A., Thompson, B., Filippi, P., \& de Boer, B. Seeking temporal predictability in speech: comparing statistical approaches on 18 world languages. Front. Hum. Neurosci. 10, 586 (2016).

47. Thorndike, R. L. Who belongs in the family? Psychometrika 18 267-276 (1953).

48. Longuet-Higgins, H. C. \& Lee, C. S. The perception of musical rhythms. Perception 11, 115-128 (1982).

49. Longuet-Higgins, H. C. \& Lee, C. S. The rhythmic interpretation of monophonic music. Music Percept. 1, 424-441 (1984).

\section{Acknowledgements}

A.R. was supported by Fonds Wetenschappelijk Onderzoek Vlaanderen grant no. V439315N, and European Research Council (ERC) grant (283435 ABACUS, to B. de Boer). The funders had no role in study design, data collection and analysis, decision to publish, or preparation of the manuscript. We thank P. Filippi, B. Thompson, B. de Boer, H. Little, S. van der Ham, N. Chr. Hansen, J. Iversen, D. Bowling, T. Grossi, A.C. Miralles, P. Norton, V. Spinosa, Y.-H $\mathrm{Su}, \mathrm{P}$. Tinits and K. Smith, as well as all members of the Centre for Language Evolution (Edinburgh), AI-Lab (VUB Brussels), Biolinguistics (Barcelona) and attendants of Evolang XI, IBAC XXV, Statistical Learning 2015 and the DZG Graduate Meeting 2016 for their comments and advice.

\section{Author contributions}

A.R. and S.K. conceived the study. A.R., T.D. and S.K. designed research.

T.D. performed the research. A.R. and S.K. wrote the Python scripts for the data analysis and experimental testing. A.R., T.D. and S.K. analysed the data and wrote the paper.

\section{Additional information}

Supplementary information is available for this paper.

Reprints and permissions information is available at www.nature.com/reprints. Correspondence and requests for materials should be addressed to A.R.

How to cite this article: Ravignani, A, Delgado, T. \& Kirby, S. Musical evolution in the lab exhibits rhythmic universals. Nat. Hum. Behav. 1, 0007 (2016).

\section{Competing interests}

The authors declare no competing interests. 